\title{
Problemática de estudiantes de desarrollo de negocios área mercadotecnia ante la pandemia
}

\section{Problematic of business development in the marketing area students in the face of the pandemic}

\author{
GUTIÉRREZ-ZEPEDA, Martha del Pilar†* \& GARCÍA-BAHENA, María Gabriela \\ Universidad Tecnológica de Nezahualcóyotl, Cuerpo Académico Nuevas Tecnologías y MIPYMES, México.
}

ID $1^{\text {er }}$ Autor: Martha del Pilar, Gutiérrez-Zepeda / ORC ID: 0000-0003-0256-8874

ID $1^{\text {er }}$ Coautor: María Gabriela, García-Bahena / ORC ID: 0000-0002-8141-5894

DOI: $10.35429 / J P D .2020 .11 .4 .8 .15$

Recibido 15 de Enero, 2020; Aceptado 30 de Junio, 2020

\section{Resumen}

A nivel mundial 2020 ha sido un año de grandes retos, debido a la pandemia desencadenada por el COVID-19, cada país ha tenido que afrontar los retos que como consecuencia se han presentado, diversos ámbitos se vieron afectados por tal situación y uno de ellos fue el educativo y aunque no podemos decir que sea el más importante si es un ámbito que tiene una repercusión muy amplia, desde la propia actividad escolar de docentes, alumnos y administrativos del centro escolar, hasta la actividad en el núcleo familiar, aunque las figuras del centro escolar han incidido debido a las circunstancias a un ambiente virtual para realizar y dar cumplimiento a todas las actividades inherentes al proceso de enseñanza aprendizaje, la dinámica familiar en esta adaptación de la escuela se ha visto afectada de forma importante, entre otras situaciones al reunir en un mismo espacio en casa a estudiantes de diversos niveles educativos para poder conectarse a sus sesiones en línea. Sin embargo, en esta adaptación se han presentado diversas dificultades que limitan el desarrollo educativo de muchos de los estudiantes y que tiene que ver con la disposición de los recursos que tienen a su alcance para poder llevar a cabo se labor educativa de la mejor manera y alcanzar adecuadamente los aprendizajes esperados y el logro de los objetivos educativos, dichas dificultades tienen relación con la inequidad económica y tecnológica. En ese sentido y con cerca de nueve meses en esta situación, es que surge la inquietud de conocer las dificultades en cuestión de recursos tecnológicos que han tenido los estudiantes de la Universidad Tecnológica de Nezahualcóyotl, en particular de la carrera de Desarrollo de Negocios Área Mercadotecnia y que han afectado su rendimiento escolar, a través de la realización de una investigación cuantitativa con una muestra aplicada a 283 alumnos de esta División, apoyados en un cuestionario. Con los resultados, se pretende contar con elementos para tomar decisiones respecto a estrategias que coadyuven a un mejor rendimiento escolar y a disminuir la brecha tecnológica de los alumnos de la Universidad Tecnológica de Nezahualcóyotl.

Pandemia, Educación, Tecnología, Aprendizaje, Rendimiento escolar

\begin{abstract}
Globally 2020 has been a year of great challenges, due to the pandemic triggered by COVID-19, each country has had to face the challenges that have arisen as a result of it, various areas were affected because of this situation and one of them was education although we cannot say that it is the most important if it is an area that has a great impact, from the own school activity of teachers, students and administrators, to the activity in the family nucleus, although the school figures have changed due to the circumstances to a virtual environment to carry out and fulfill all the activities inherent in the learning teaching process, the family dynamics in this adaptation of the school have been significantly affected, among other situations by bringing together students of various educational levels in the same space at home in order to connect to their online sessions. However, this adaptation has presented a few difficulties that limit the educational development of many of the students and which has to do with the provision of the resources available to them to carry out educational work in the best way and properly achieve the expected learnings and the achievement of educational objectives, these difficulties relate to economic and technological inequity. In this sense and with about nine months in this situation, it is concerned to know the difficulties in terms of technological resources that students of the Technological University of Netzahualcoyotl have had, in particular of the carreer of Business Development Marketing Area and that have affected their school performance, through the conduct of quantitative research with a sample applied to 283 students of this area, supported by a questionnaire. With the results, it is intended to have elements to make decisions regarding strategies that contribute to better school performance and to reduce the technological gap of students at the Technological University of Netzahualcoyotl.
\end{abstract}

Citación: GUTIÉRREZ-ZEPEDA, Martha del Pilar \& GARCÍA-BAHENA, María Gabriela. Problemática de estudiantes de desarrollo de negocios área mercadotecnia ante la pandemia. Revista de Didáctica Práctica. 2020. 4-11:8-15.

*Correspondencia al Autor (Correo Electrónico: martha.gutierrezze@ utn.edu.mx)

$\dagger$ Investigador contribuyendo como primer autor. 


\section{Introducción}

La situación emergente en la que nos encontramos por la pandemia ocasionada por el COVID-19 nos hace buscar alternativas de adaptación que en muchos de los casos son emergentes e improvisadas y a partir de lo que conocemos por haberlo realizado anteriormente, tal es el caso de la actividad educativa, alumnos $\mathrm{y}$ docentes ante la emergencia tuvieron que adaptar la educación en línea a partir de su experiencia en la educación presencial, sin mayor análisis que la problemática que se vivía por la pandemia, sin embargo para muchos no fue la mejor experiencia ni se dieron los mejores resultados.

Por lo anterior, el proyecto de investigación que se detalla a continuación se desarrolló con el fin de saber que tanto afecto la actual pandemia causada por el COVID 19 en el rendimiento e interés de los estudiantes en sus clases, de la carrera de Desarrollo de Negocios Área Mercadotecnia de la Universidad Tecnológica de Nezahualcóyotl (UTN), ya que el simple hecho de estudiar a distancia o en línea resultó ser un gran reto, así como un cambio inesperado para la comunidad estudiantil de la UTN y de todo el sistema educativo de México.

En tal sentido, el presente documento integra preponderantemente los resultados de una investigación realizada a los alumnos en comento, que permita identificar las dificultades que en cuestión tecnológica y de interés han tenido en la modalidad educativa a distancia por la que se tuvo que optar, integrando para ello un análisis inicial de la situación, el desarrollo metodológico llevado a cabo para la obtención de información, los resultados emanados y, por último, las conclusiones y sugerencias que se derivan de los hallazgos encontrados.

\section{Metodología}

\section{Planteamiento del Problema}

La pandemia ocasionada por el COVID-19 ha provocado una situación sin precedentes en todos los ámbitos de la vida. El confinamiento obligado como consecuencia, ha afectado a todos los niveles educativos.
Suspender las actividades de enseñanza aprendizaje presenciales ocasionó que se trasladaran como medida emergente a un modelo virtual en el que el hecho de adaptarse a esa nueva modalidad de estudio resultara para algunos estudiantes y docentes bastante caótico, muchos no están acostumbrados a la digitalización y/o a la comunicación virtual, aunado a que hay una mayoría desfavorecida (por lo menos en México) que no cuenta con los suficientes recursos para el aprendizaje en línea, de tal forma, que resulta en un gran obstáculo para el logro de los objetivos educativos que cada quien tiene.

El desafío hoy en día de las universidades sobretodo públicas (y de todo el sistema educativo nacional), tiene varios pendientes por revisar; uno puede ser, las limitantes que el propio sistema en línea conlleva; otro, la falta de habilidades de los profesores para tener competencias en el manejo de ambientes virtuales para dirigir un aprendizaje exitoso; y en el caso de los jóvenes, se presentan dificultades muy similares, pero sobretodo en la falta de competencias digitales para auto aprender, para trabajar en ambientes de aprendizaje que impliquen la falta de cercanía física con el profesor, y la falta de herramientas e instrumentos para realizar trabajos virtuales. Respecto a las últimas limitantes mencionadas en el párrafo anterior, se considera como la principal afectación en los estudiantes de la UTN por el que el rendimiento académico se ha visto notoriamente afectado, motivo por el cual se desea conocer más a fondo cuales son los recursos principales que han afectado el buen desempeño académico en los últimos meses.

\section{Marco Referencial}

"La UNESCO estima que el cierre de las instituciones educativas debido a la pandemia está afectando a la mitad de los estudiantes del mundo. La enseñanza en línea es ahora una rutina nueva para algunos estudiantes, pero presenta desafíos importantes. No todos los estudiantes tienen acceso a este tipo de educación, considerando la desigualdad social." (Gonzáles Hernández, 2020); lo anterior es más evidente en México en instituciones de educación pública incluyendo las de nivel superior, aunque las universidades están más preparadas para dicha modalidad al contar en su mayoría con planes de estudios a distancia, la desigualdad social también está presente.

GUTIÉRREZ-ZEPEDA, Martha del Pilar \& GARCÍABAHENA, María Gabriela. Problemática de estudiantes de desarrollo de negocios área mercadotecnia ante la pandemia. Revista de Didáctica Práctica. 2020 
"En la educación en línea algunas familias afrontan también las dificultades de conexión. La educación online, mediante plataformas o los programas en canales de televisión o radio, no son posibles siempre por problemas de acceso." (UNIR, 2020); problemas derivados principalmente de factores económicos y sociales.

La desigualdad social y educativa que puede profundizarse en las circunstancias actuales y que se materializa en la brecha de acceso a las tecnologías según el nivel socioeconómico de las familias, así como en las propias trayectorias educativas de los padres que hacen diferencia en cuanto al apoyo que pueden dar a sus hijos; en efecto, "no todos parten del mismo punto ni tienen los mismos recursos o capacidades para hacerlo de manera efectiva." (Ruíz Cuellar, 2020); otro aspecto importante a considerar en la brecha de desigualdad tecnológica lo son el tamaño y composición de la familia, en ocasiones los padres de familia le "tienen" que dar prioridad a las necesidades y requerimientos de estudio de alguno de sus integrantes sobre otros ante la carencia de recursos para cubrir las necesidades de todos los integrantes.

"Será un gran desafío para el sistema educativo nacional, porque la falta de conectividad en el país, la carencia de dispositivos entre los estudiantes y la escasa capacitación de los docentes en el desarrollo de habilidades tecnológicas, van a limitar el aprendizaje, pero al mismo tiempo nos va hacer acelerar el modelo de enseñanza digital que ya venía proyectándose desde hace años." (Flores Leal, 2020); aun a pesar de las desafiantes dificultades que se vislumbran, se debe reconocer que la actual situación educativa representa una gran oportunidad para fortalecer la entrada ya inminente de la educación a distancia que como se comenta se estaría adelantando a su tiempo.

\section{Objetivo General}

Identificar cuáles son los recursos que afectaron el desempeño escolar de los estudiantes de TSU en Desarrollo de Negocios Área Mercadotecnia en la UTN a través de una recolección de datos (encuesta) durante la pandemia de COVID-19 en un tiempo determinado de 2 a 3 meses buscando encontrar una posible solución para que el desempeño de los estudiantes mejore.

\section{Diseño de la Investigación}

Se desarrolló una Investigación descriptiva con un enfoque cuantitativo que permita a través de un análisis cuantificable conocer los recursos que afectaron el desempeño escolar de los estudiantes de la UTN.

\section{Método de Recolección e Instrumento}

Se decido utilizar el método de encuesta para la recolección de información, para lo cual se diseñó un instrumento (cuestionario) de preguntas con respuesta cerrada, que permitió obtener la información necesaria respecto a los recursos que limitaron el desempeño académico de los estudiantes de la UTN y se integró de la siguiente manera:

\section{INSTRUMENTO DE RECOLECCIÓN DE DATOS FINAL}

Universidad Tecnológica de Nezahualcóyotl Desarrollo de Negocios Área Mercadotecnia Tema: "De qué manera influye la actual situación en el desempeño escolar de los jóvenes"

Edad: Sexo:

1 ¿Con que aparato electrónico cuentas para tomar tus clases en línea?

a. Teléfono celular b. Computadora c. Tablet d. Ninguno

2. ¿Desde qué lugar te conectas para tomar tus clases?

a. Café internet b. Trabajo c. Casa de algún familiar con internet d. En lugares públicos con internet gratis e. En mi hogar

3. ¿Has solicitado ayuda con tus compañeros para poder realizar tus trabajos en línea?

a. Si b. A veces c. La mayoría de las veces d. No

4. Para tomar tus clases te conectas por medio de:

a. Datos b. Internet

5. ¿De qué manera te ha afectado la falta de herramientas para tomar tus clases en línea?

GUTIÉRREZ-ZEPEDA, Martha del Pilar \& GARCÍABAHENA, María Gabriela. Problemática de estudiantes de desarrollo de negocios área mercadotecnia ante la pandemia. Revista de Didáctica Práctica. 2020 
a. En mis calificaciones b. En mi aprendizaje c. En mis trabajos d. En mis faltas

6. ¿Consideras que está nueva modalidad te ha permitido aprender y obtener nuevos conocimientos?

a. Siempre b. A veces c. La mayoría de las veces d. Para nada

7. ¿Cómo te sientes emocionalmente tomando clases a distancia sin tener los medios electrónicos?

a. Frustrado b. Preocupado c. Desesperado d. Desmotivado

8. ¿Obtienes ayuda por parte de los profesores cuando no te es posible asistir a tus clases virtuales?

a. Siempre b. A veces c. Casi siempre d. Nunca

9. ¿Sientes la necesidad de abandonar tus clases virtuales por la falta de medios y aprendizaje?

a. Definitivamente b. A veces c. Lo he pensado d. Para nada

10. ¿Consideras que la institución toma en cuenta a los alumnos que no cuentan con los elementos necesarios para implementar posibles estrategias y soluciones para ellos?

\section{a. Si b. No}

Le agradecemos su colaboración y tiempo que nos brinda para este proyecto de investigación. ¡Que tenga un excelente día!

\section{Universo y Muestra}

Se trabajó con una población finita de 773 alumnos de la carrera de Desarrollo de Negocios Área Mercadotecnia y a través del uso de la fórmula de poblaciones finitas se obtuvo una muestra finita de 257 elementos (aunque nos contestaron un poco más de esa cantidad, 283 alumnos, la cual decidimos manejar por ser aproximada a la muestra obtenida), manejando un nivel de confianza de $95 \%$ y un margen de error de $5 \%$.

\section{Recolección de Datos}

La recolección de datos se realizó a través la plataforma TEAMS durante los días 5, 6 y 7 de octubre del 2020 mediante un formulario en FORMS, con la colaboración de alumnos de cuarto cuatrimestre de la División de Comercialización.

\section{Análisis de los Datos}

El análisis de datos fue estadístico, y la información obtenida se tabuló y graficó, adicionando los respectivos análisis e interpretación de resultados.

\section{Resultados}

De acuerdo con la aplicación del cuestionario a los estudiantes de la Universidad Tecnológica de Nezahualcóyotl de la carrera Desarrollo de Negocios área Mercadotecnia, se obtuvieron los siguientes resultados.

\section{Hombre Mujer $56 \%$ 44\%}

Tabla 1 Género

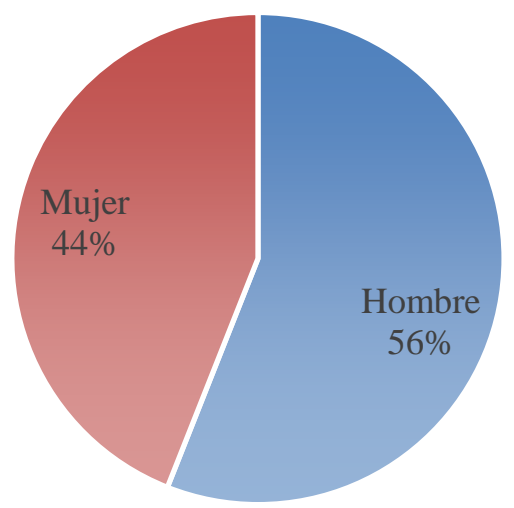

\section{Gráfico 1}

Fuente: Elaboración propia

Como se muestra en la gráfica se encuestaron más hombres que mujeres.

\begin{tabular}{|l|r|}
\hline \multicolumn{1}{|c|}{ Dispositivos } & \multicolumn{1}{c|}{$\%$} \\
\hline Teléfono & $60 \%$ \\
\hline Computadora & $31 \%$ \\
\hline Tablet & $5 \%$ \\
\hline Ninguno & $4 \%$ \\
\hline
\end{tabular}

Tabla 2 ¿Con qué aparato electrónico cuenta para tomar clases en línea? 


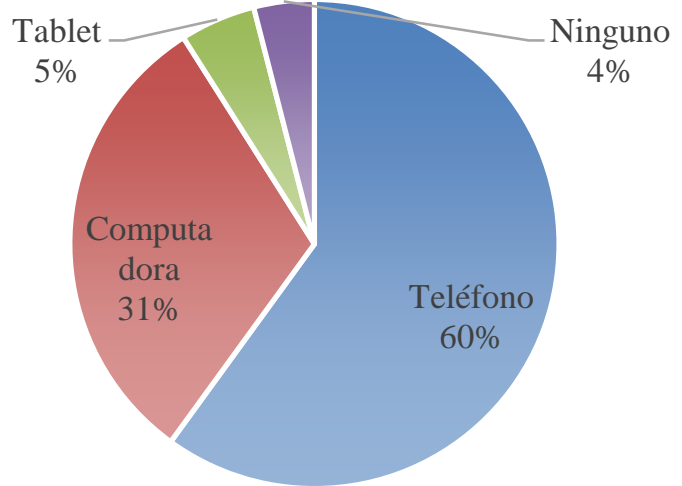

Gráfico 2

Fuente: Elaboración propia

Uno de los recursos más utilizados en esta Institución fue el teléfono y le sigue la computadora para la realización de sus actividades académicas.

\begin{tabular}{|l|c|}
\hline \multicolumn{1}{|c|}{ Lugar } & $\mathbf{\%}$ \\
\hline Café internet & $4 \%$ \\
\hline Trabajo & $10 \%$ \\
Casa con un familiar que tiene internet. & $27 \%$ \\
\hline Lugar público con internet. & $17 \%$ \\
\hline En mi hogar & $42 \%$ \\
\hline
\end{tabular}

Tabla 3 ¿Desde qué lugar se conecta para tomar clases?

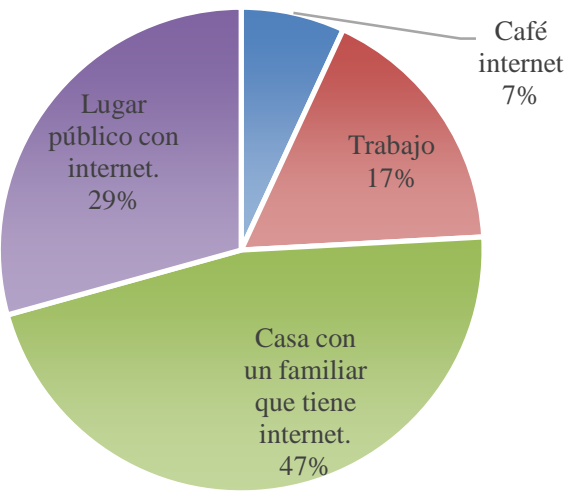

Gráfico 3

Fuente: Elaboración propia

En el gráfico se muestra que la mayoría si tiene internet en un $42 \%$, pero también es preocupante que el $27 \%$ de los alumnos necesitan acudir con alguien más para tomar clases.

\begin{tabular}{|c|c|}
\hline $\mathrm{Si}$ & No \\
\hline $55 \%$ & $45 \%$ \\
\hline
\end{tabular}

Tabla 4 ¿Ha solicitado ayuda con sus compañeros para poder realizar sus trabajos en línea?

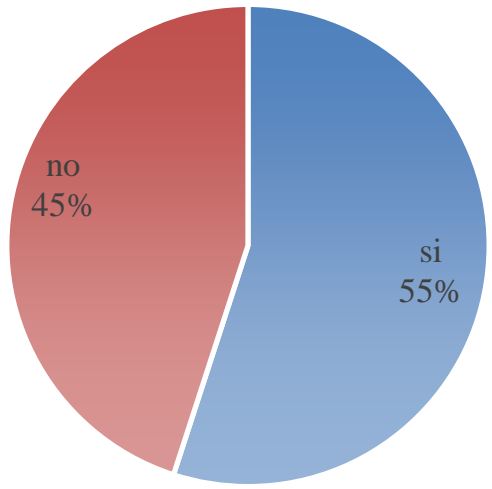

Gráfico 4

Fuente: Elaboración propia

Como se muestran los resultados de la gráfica la mayoría de los alumnos si piden ayuda a sus compañeros para elaborar sus trabajos.

\begin{tabular}{|l|r|}
\hline \multicolumn{1}{|c|}{ Afectaciones } & \% \\
\hline En calificaciones & $49 \%$ \\
\hline En el aprendizaje & $31 \%$ \\
\hline En las tareas & $17 \%$ \\
\hline En faltas & $3 \%$ \\
\hline
\end{tabular}

Tabla 5 ¿De qué manera le ha afectado la falta de herramientas para tomar las clases en línea?

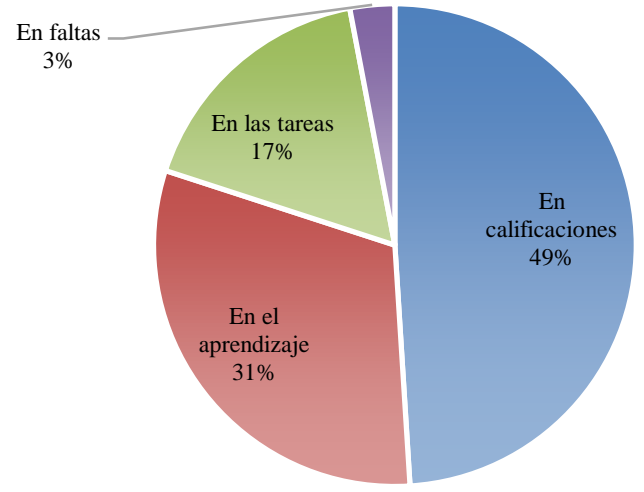

\section{Gráfico 5}

Fuente: Elaboración propia

Como se muestra en este gráfico una de las afectaciones que se considera la principal como estudiante son las calificaciones en un $49 \%$ que es en su gran mayoría al no tener las herramientas adecuadas para su estudio.

\begin{tabular}{|l|r|}
\hline \multicolumn{1}{|c|}{ Frecuencia } & \% \\
\hline Siempre & $6 \%$ \\
\hline A veces & $35 \%$ \\
\hline La mayoría de las veces & $33 \%$ \\
\hline Nada & $26 \%$ \\
\hline
\end{tabular}

Tabla 6 ¿Considera que esta nueva normalidad ha permitido aprender y obtener nuevos conocimientos?

GUTIÉRREZ-ZEPEDA, Martha del Pilar \& GARCÍABAHENA, María Gabriela. Problemática de estudiantes de desarrollo de negocios área mercadotecnia ante la pandemia. Revista de Didáctica Práctica. 2020 


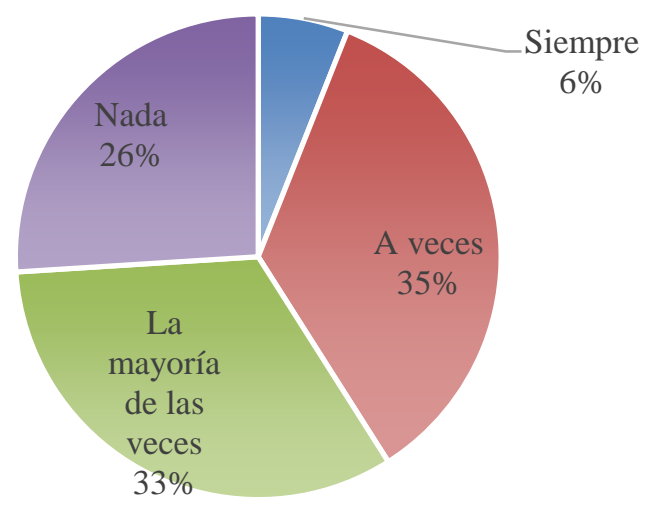

Gráfico 6

Fuente: Elaboración propia

Como se muestra en la representación gráfica nos indica que, si aprenden la mayoría de las veces, eso indica que a pesar de que el modelo es escolarizado esta carrera si es posible trabajarla en línea.

\begin{tabular}{|l|c|}
\hline \multicolumn{1}{|c|}{ Emociones } & $\%$ \\
\hline Frustrado & $55 \%$ \\
\hline Preocupado & $21 \%$ \\
\hline Desesperado & $12 \%$ \\
\hline Desmotivado & $12 \%$ \\
\hline
\end{tabular}

Tabla 7 ¿Cómo se siente emocionalmente tomando clases a distancia sin tener los medios electrónicos?

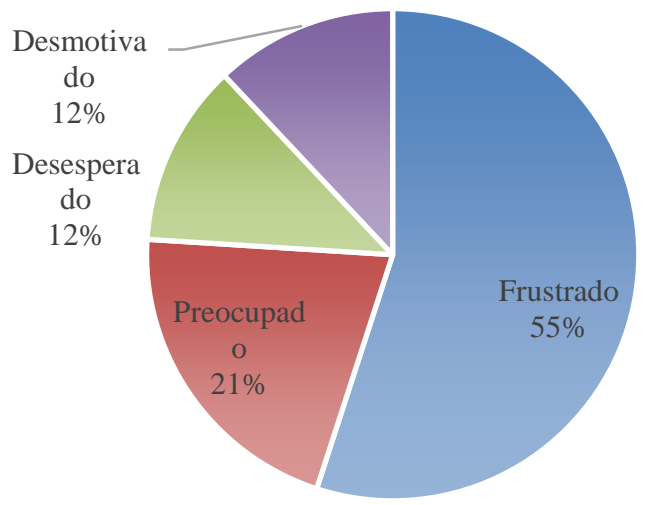

Gráfico 7

Fuente: Elaboración propia

Como se muestra en esta tabla nos indica que el alumno cuando no tiene los medios adecuados para el estudio la mayoría se siente frustrado.

\begin{tabular}{|l|c|}
\hline Frecuencia & \% \\
\hline Siempre & $11 \%$ \\
\hline A veces & $39 \%$ \\
\hline Casi nunca & $31 \%$ \\
\hline Nunca & $19 \%$ \\
\hline
\end{tabular}

Tabla 8 ¿Obtiene ayuda de sus profesores cuando no es posible permanecer en la clase virtual?

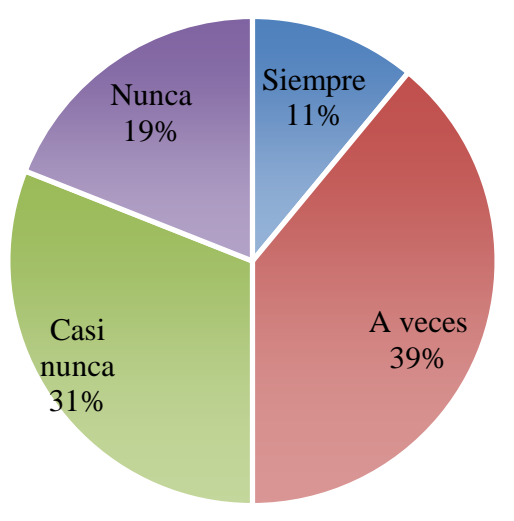

\section{Gráfico 8}

Fuente: Elaboración propia

Como se muestra en la figura el 39\% si tiene algún apoyo por parte del profesor.

\begin{tabular}{|l|c|}
\hline \multicolumn{1}{|c|}{ Frecuencia } & $\%$ \\
\hline Definitivamente & $47 \%$ \\
\hline A veces & $23 \%$ \\
\hline Lo he pensado & $13 \%$ \\
\hline Nunca & $17 \%$ \\
\hline
\end{tabular}

Tabla 9 ¿Siente la necesidad de abandonar las clases virtuales por la falta de medios y aprendizaje?

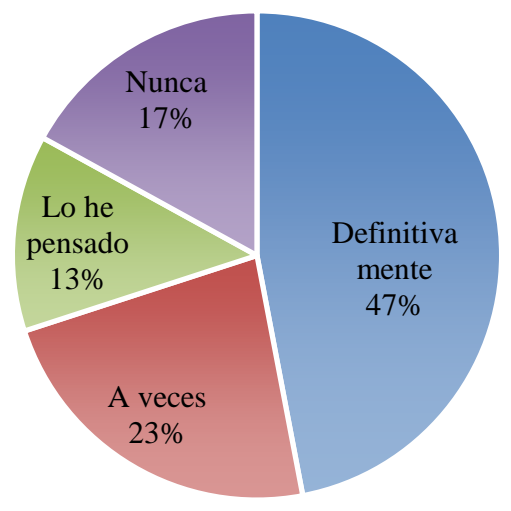

\section{Gráfico 9}

Fuente: Elaboración propia

Casi la mitad de los alumnos que no tienen los recursos adecuados (el 4\%) de ellos para el estudio en línea, indican que, si se saliesen de la Universidad, sin embargo, de acuerdo a las estadísticas registradas de este cuatrimestre el $1 \%$ de la población en aula desertó por diferentes situaciones (Informe cuatrimestral del PIT 2020-3)

\begin{tabular}{|c|c|}
\hline $\mathbf{S i}$ & $\mathbf{N o}$ \\
\hline $45 \%$ & $55 \%$ \\
\hline
\end{tabular}

Tabla 10 ¿Considera que la Institución toma en cuenta a los alumnos que no cuentan con los elementos necesarios para implementar las posibles estrategias o soluciones? 


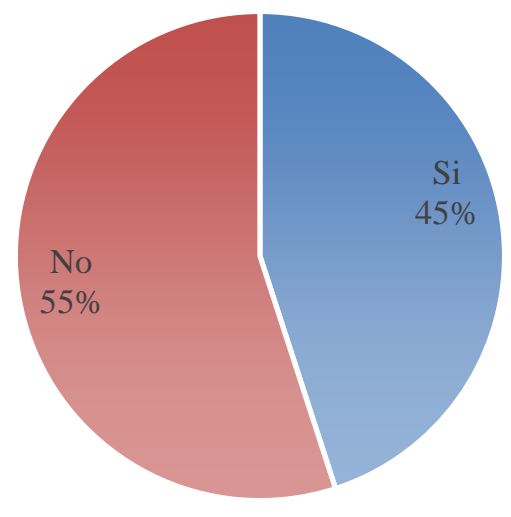

Gráfico 10

Fuente: Elaboración propia

La mayoría de los estudiantes tienen un panorama negativo al respecto, tienen la percepción de que la institución no los apoya.

\section{Conclusiones}

De acuerdo con los resultados se concluye lo siguiente:

1. Los recursos que más utilizan los estudiantes de la Universidad Tecnológica de Nezahualcóyotl son en un $60 \%$ el teléfono celular y como segundo lugar la computadora con un $31 \%$ esto nos dice que el teléfono es una de las herramientas más utilizadas, porque en casa en la gran mayoría tienen solo un equipo de cómputo y es para toda la familia, por lo que es difícil que tenga una personal y por ello el $55 \%$, es decir un poco más de la mitad de los estudiantes les piden a sus compañeros la ayuda para la realización de sus actividades académicas.

2. Casi la mitad de los alumnos se conectan dentro de su hogar esto les proporciona una gran ventaja debido a las excesivas cargas de actividades que en ocasiones se presentan. Pero en un $27 \%$ de los estudiantes nos dicen que deben de acudir con un familiar para poder conectarse a las clases y esto impacta en las distracciones que puedan tener según los entrevistados.

3. El $49 \%$ de los estudiantes informan que les ha afectado el no tener las herramientas adecuadas en sus calificaciones y por esto poco más de la mitad (el 55\%) se siente frustrado ante esta situación.
La planeación didáctica del próximo cuatrimestre deberá realizarse tratando de elegir estrategias de aprendizaje que sean posible manejar aun en smartphone de forma práctica, alternativas de interacción asincrónicas y un mayor acercamiento vía chats o videochats con los alumnos para conocer de propia voz sus inquietudes.

\section{Propuestas}

1. La Universidad Tecnológica de Nezahualcóyotl tomó la determinación de trabajar en la Plataforma TEAMS misma que antes de iniciar clases impartió un diplomado a todos sus maestros en relación con el manejo de esta y de acuerdo con cada carrera los directores consideraron lo mejor para sus estudiantes. en este caso fue las clases online y grabadas de acuerdo con el horario establecido para que las vieran en el momento que fuese requerido para su aprendizaje.

Por lo anterior se propone que la asistencia la tomen en el momento que puedan ver los alumnos la clase para que no omitan algún tema de importancia y baje su rendimiento.

2. En esta casa de estudios el 27 de octubre de este mismo año, lanzó una convocatoria para evitar el abandono escolar por problemas derivados por la pandemia, se otorgaron 290 becas para esta noble causa, la ayuda consistía ayuda económica para la compra de un equipo de cómputo o que no contaran con servicio de internet domiciliario y/o que no podían pagar la Inscripción o Reinscripción para su educación. Esta se difundió por medio de tutores de cada uno de los grupos, así como en la página de la Universidad.

Por lo anterior se propone que los estudiantes les den seguimiento a la ayuda que se proporciona, debido que no hubo mucha participación en este programa único.

3. Se propone que los tutores sensibilicen a los alumnos de esta nueva normalidad y la importancia de tomar las clases en línea debido a las medidas de seguridad que nos indican las autoridades. 
4. Es importante que los Profesores crean sesiones de asesoría (grabando estas asesorías) de algún tema en especial que consideran que es difícil de aprender para que haya una mejor interactividad con los alumnos.

5. Que haya retroalimentaciones grabadas (Podcast) para que los alumnos puedan aclarar dudas en cualquier momento que realicen sus actividades académicas.

6. Que se calendaricen las actividades con fechas más abiertas para que tengan tiempo de planear en que momento realizaran sus actividades los alumnos y no se sientan frustrados porque sabrán cuanto tiempo tendrán para entregar las tareas asignadas.

\section{Agradecimientos}

Agradecemos de manera especial el apoyo que siempre hemos recibido de parte de todos y cada uno de los colaboradores del cuerpo académico Nuevas Tecnologías y MIPyMES, a la Universidad Tecnológica de Nezahualcóyotl por permitirnos realizar una actividad sustantiva en nuestra labor docente como lo es la investigación a los alumnos Castillo Castillo Marion Jazmín, Gallegos Márquez Jacqueline Elizbeth, León Zaragoza Alejandra Edith, Licona Sánchez Jessica Jatziri y Pio Segundo María Fernandez, mismos que fueron parte primordial en la elaboración del instrumento y la recolección de datos para esta investigación y por último a ECORFAN-México, S.C. por brindarnos la posibilidad de poder exponer el trabajo realizado.

\section{Referencias}

Flores Leal, P. (02 de septiembre de 2020). El Economista. Recuperado el Diciembre de 2020, de

https://www.eleconomista.com.mx/arteseideas/ Falta-de-conectividad-y-dispositivos-limitan-elaprendizaje-online-20200902-0106.html

Gonzáles Hernández, W. (Julio de 2020). Transformaciones de la Carrera Licenciatura en Educación Informática para disminuir las afectaciones por la Covid. Recuperado el diciembre de 2020, de https://www.researchgate.net/profile/Walfredo_
Gonzalez_Hernandez/publication/344476648_T ransformaciones_de_la_Carrera_Licenciatura_e n_Educacion_Informatica_para_disminuir_las_ afectaciones_por_la_Covid/links/5f7b2243a6fd cc00865748c8/Transformaciones-de-la-

Carrera-Licenciatura-en-Educacion-

Informatica-para-disminuir-las-afectacionespor-la-Covid.pdf

Miguel Roman, J. A. (Julio de 2020). La educación superior en tiempos de pandemia: una visión desde dentro del proceso formativo. Recuperado el Diciembre de 2020, de Redalyc: https://www.redalyc.org/jatsRepo/270/2706323 7017/html/index.html

Ruíz Cuellar, G. (Agosto de 2020). Covid-19: pensar la educación en un escenario inédito. Recuperado el Diciembre de 2020, de Revista mexicana de investigación educativa (SciELO): http://www.scielo.org.mx/scielo.php?script=sci _arttext\&pid=S1405-66662020000200229

UNIR. (Julio de 2020). Cómo ha afectado a la educación la pandemia por COVID-19. Recuperado el Diciembre de 2020, de UNIR la universidad en internet: https://mexico.unir.net/vive-unir/como-haafectado-a-la-educacion-la-pandemia-porcovid-19/

Villafuerte, P. (Mayo de 2020). El aprendizaje remoto enfrenta otro reto: el profesorado no esta preparado para la enseñanza en línea. Recuperado el Diciembre de 2020, de Observatorio de Onovación Educativa: https://observatorio.tec.mx/edunews/profesorado-no-esta-preparado-paraeducacion-online

Villafuerte, P. (Marzo de 2020). Educación en tiempos de pandemia: COVID-19 y equidad en el aprendizaje. Recuperado el Diciembre de 2020, de Observatorio de Inovación Educativa: https://observatorio.tec.mx/edunews/educacion-en-tiempos-de-pandemiacovid19 